\title{
DESENVOLVIMENTO DE SOFTWARE EDUCACIONAL PARA DIMENSIONAMENTO DE VIGAS E LAJES EM CONCRETO ARMADO
}

\author{
DEVELOPMENT OF EDUCATIONAL SOFTWARE FOR THE DESIGN OF \\ REINFORCED CONCRETE BEAMS AND SLABS
}

\author{
Gabriel Antonio Neto - gabriel.antonio292@gmail.com \\ Universidade de Araraquara (UNIARA) - Araraquara -SP - Brasil \\ Fabiana Florian - fflorian@uniara.edu.br \\ Universidade de Araraquara (UNIARA) - Araraquara -SP - Brasil \\ José Eduardo Quaresma - jequaresma@uniara.com.br \\ Universidade de Araraquara (UNIARA) - Araraquara -SP - Brasil
}

DOI: 10.31510/infa.v17i2.873

Data de publicação: 18/12/2020

\section{RESUMO}

O objetivo principal deste trabalho é apresentar um software livre para microcomputadores de fácil compreensão e interface amigável, a fim de resolver numericamente o problema de dimensionamento de vigas e lajes, desde o pré-dimensionamento até as armaduras, longitudinais e estribos, mostrando graficamente e numericamente o momento fletor e cortante em vigas tendo como base a norma brasileira NBR 6118-2014. Tal aplicativo, denominado Orion, foi desenvolvido com o objetivo de facilitar o entendimento dos alunos em matérias de cálculos estruturais de concreto armado, sendo utilizado apenas para fins acadêmicos, mostrando de forma simples e rápida os resultados obtidos. O conceito de software livre não é muito difundido no Brasil, quando se trata de software livre para engenharia civil há grande dificuldade em encontrar bons programas, afinal software livre é um programa de computador distribuído sob condições que permite aos usuários estudá-lo, modificá-lo e distribuí-lo. $\mathrm{O}$ software é considerado livre se fornecer ao usuário o controle final sobre o código-fonte do programa, permitindo sua modificação e posterior distribuição. Para o desenvolvimento do Orion foi utilizada a linguagem de programação JAVA, a fim de maximizar seu alcance tornando-o multiplataforma e atingindo o maior número de pessoas.

Palavra-Chave: Concreto Armado. Viga. Laje. Momento cortante. Momento fletor.

\section{ABSTRACT}

The main objective of this work is to present free software for microcomputers that is easy to understand and friendly interface, in order to numerically solve the problem of dimensioning beams and slabs, from pre-dimensioning to reinforcement, longitudinal and stirrups, showing 
graphically and numerically the bending and cutting moment in beams based on the Brazilian standard NBR 6118-2014. This application, called Orion, was developed with the objective of facilitating students' understanding of structural calculations in reinforced concrete, being used only for academic purposes, showing the results obtained in a simple and quick way. The concept of free software is not widespread in Brazil, when it comes to free software for civil engineering there is great difficulty in finding good programs, after all free software is a computer program distributed under conditions that allows users to study it, modify it it and distribute it. The software is considered free if it provides the user with final control over the source code of the program, allowing its modification and subsequent distribution. For the development of Orion, the JAVA programming language was used in order to maximize its reach by making it multiplatform and reaching the largest number of people.

Keywords: Reinforced concrete. Beam. Slab. Cutting moment. Bending moment.

\section{INTRODUÇÃO}

Concreto é um material de construção proveniente da mistura, em proporção adequada, de: aglomerantes, agregados e água. Também é frequente o emprego de aditivos e adições. (PINHEIRO, 2010).

Com o crescente avanço digital, o uso de tecnologias está cada vez mais presente no cotidiano das pessoas, na engenharia não diferentes, para a realização de cálculos avançados ou projetos complexos, é necessário o uso de softwares. Embora grande parte deles esteja localizada no domínio privado, ou seja, uma empresa ou instituição possui uma licença de distribuição, na engenharia civil a variedade de softwares livres com finalidade de estudos é escassa.

Em meio à falta de opções de softwares para engenharia, aumenta a necessidade de elaboração de um software livre focado na solução de problemas didáticos relacionados a matéria de concreto armado, com base na norma brasileira NBR 6118-2014 projeto de estruturas de concreto - Procedimento. Dito isso, o objetivo principal deste trabalho é apresentar um software livre para microcomputadores de fácil compreensão e interface amigável, a fim de resolver numericamente o problema de dimensionamento de vigas e lajes.

\section{DOMÍNIOS DE CÁLCULOS}

As principais propriedades mecânicas do concreto são: resistência à compressão, resistência à tração e módulo de elasticidade. Essas propriedades são determinadas a partir de ensaios, executados em condições específicas. (PINHEIRO, 2010). 
A formulação do Método dos Elementos Finitos (MEF) requer a existência de uma equação integral, de modo que seja possível substituir o integral sobre um domínio complexo (de volume V) por um somatório de integrais estendidos a sub domínios de geometria simples (de volume Vi). (AZEVEDO, 2003).

As configurações possíveis do diagrama de deformações correspondentes ao estado limite último para uma seção submetida a solicitações normais sugerem a delimitação de regiões, chamadas domínios de deformações, onde poderá estar contido o diagrama de deformações referente a um determinado caso de solicitação normal quando o estado limite último for atingido. (FERNANDES, 2006).

Figura 1- Capacidade resistente da seção

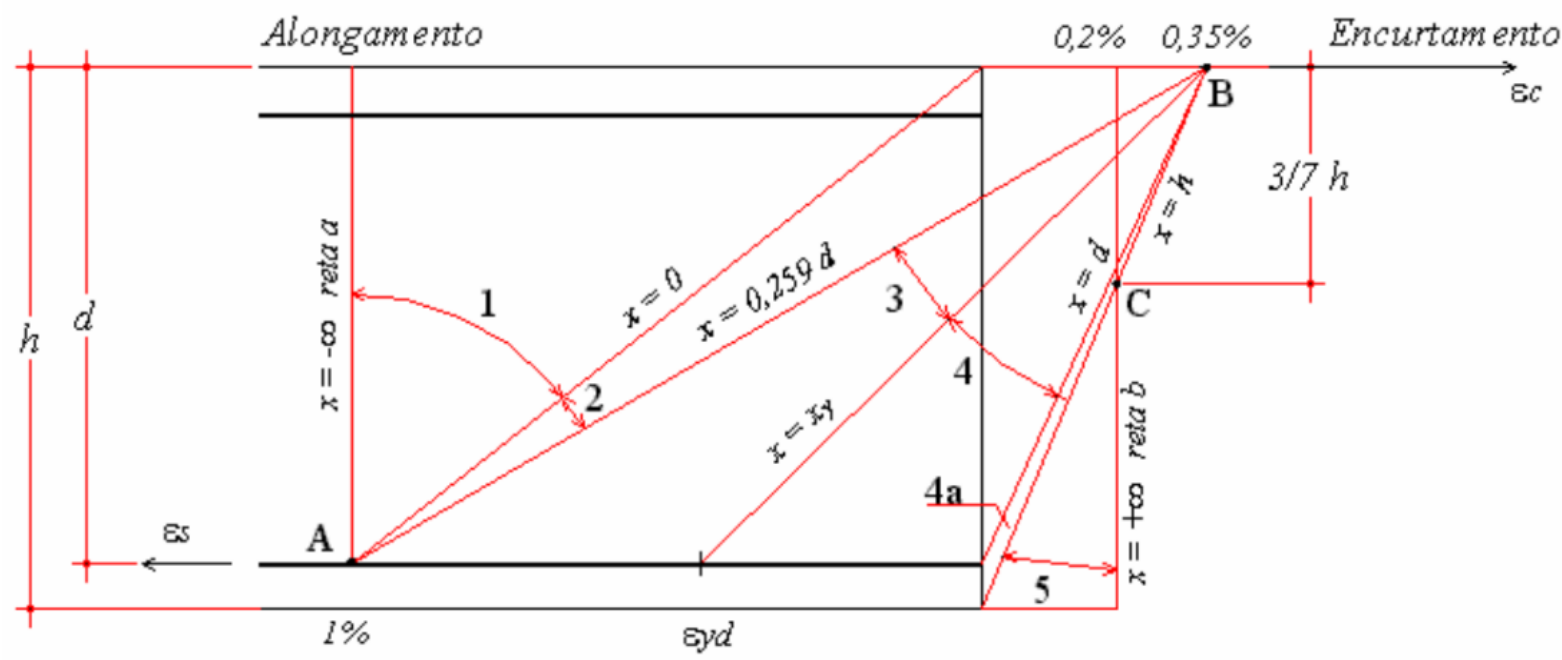

Fonte: Fernandes, 2006.

$\mathrm{Na}$ figura 1 estão representados os domínios de deformações e as retas que correspondem aos limites entre cada um deles.

- $\quad$ Domínio 1: Flexo-tração sem tensões de compressão.

- $\quad$ Domínio 2: Flexão simples/composta sem ruptura à compressão do concreto $(\mathcal{E}<3,5)$ e com alongamento permitido para as armaduras $\left(\mathcal{E s}_{\mathrm{s}}<10\right)$.

- $\quad$ Domínio 3: Flexão simples/composta com ruptura à compressão do concreto e com escoamento das armaduras $\left(\mathcal{E}_{\mathrm{s}}>\mathcal{E}_{\mathrm{yd}}\right)$.

- $\quad$ Domínio 4: Flexão simples/composta com ruptura à compressão do concreto e armaduras tracionadas sem escoamento $\left(\varepsilon_{s}>\varepsilon y d\right)$.

- $\quad$ Domínio 4a: Flexão composta com armaduras comprimidas. 
- $\quad$ Domínio 5: Flexo-compressão sem tensões de tração.

\subsection{CASSE DE AGRESSIVIDADE}

Nos projetos das estruturas correntes, a agressividade ambiental deve ser classificada de acordo com o apresentado na Quadro 1 e pode ser avaliada, simplificadamente, segundo as condições de exposição da estrutura ou de suas partes. (ABNT NBR 6118:2014).

\begin{tabular}{|c|c|c|c|c|}
\hline \multicolumn{5}{|c|}{ Quadro 1- Classes de agressividade ambiental (CAA) } \\
\hline & $\begin{array}{l}\text { Classe de } \\
\text { agressividade } \\
\text { ambiental }\end{array}$ & Agressividade & $\begin{array}{l}\text { Classificação geral do tipo de } \\
\text { ambiente para efeito de projeto }\end{array}$ & $\begin{array}{l}\text { Risco de } \\
\text { deterioração da } \\
\text { estrutura }\end{array}$ \\
\hline \multirow{2}{*}{\multicolumn{2}{|c|}{ I }} & \multirow{2}{*}{ Fraca } & Rural & \multirow{2}{*}{ Insignificante } \\
\hline & & & Submersa & \\
\hline & II & Moderada & Urbana a, b & Pequeno \\
\hline \multirow{2}{*}{\multicolumn{2}{|c|}{ III }} & \multirow{2}{*}{ Forte } & Marinha ${ }^{a}$ & \multirow{2}{*}{ Grande } \\
\hline & & & Industrial a, b & \\
\hline \multirow{2}{*}{\multicolumn{2}{|c|}{ IV }} & \multirow{2}{*}{ Muito forte } & Industrial a, c & \multirow{2}{*}{ Elevado } \\
\hline & & & Respingos de maré & \\
\hline \multicolumn{5}{|c|}{$\begin{array}{l}\text { Pode-se admitir um microclima com uma classe de agressividade mais branda (uma classe acima) para } \\
\text { ambientes internos secos (salas, dormitórios, banheiros, cozinhas e áreas de serviço de apartamentos } \\
\text { residenciais e conjuntos comerciais ou ambientes com concreto revestido com argamassa e pintura). }\end{array}$} \\
\hline b & \multicolumn{4}{|c|}{$\begin{array}{l}\text { Pode-se admitir uma classe de agressividade mais branda (uma classe acima) em obras em regiões } \\
\text { de clima seco, com umidade média relativa do ar menor ou igual a } 65 \% \text {, partes da estrutura protegidas } \\
\text { de chuva em ambientes predominantemente secos ou regiões onde raramente chove. }\end{array}$} \\
\hline c & \multicolumn{4}{|c|}{$\begin{array}{l}\text { Ambientes quimicamente agressivos, tanques industriais, galvanoplastia, branqueamento em indús- } \\
\text { trias de celulose e papel, armazéns de fertilizantes, indústrias químicas. }\end{array}$} \\
\hline
\end{tabular}

Fonte: ABNT NBR 6118:2014.

\section{SISTEMA ORION}

O programa, implementado na linguagem $\mathrm{Java}^{\circledR}$, foi desenvolvido para análise e dimensionamento de vigas e lajes. O usuário pode escolher entre calcular as Reações de apoio ou Momento fletor de uma laje, ou as armaduras de uma viga.

\subsection{Janela Principal}

A janela principal do software, Figura 2; é minimalista, de modo que o usuário escolha o cálculo que será realizado, por meio do menu "Programas". 


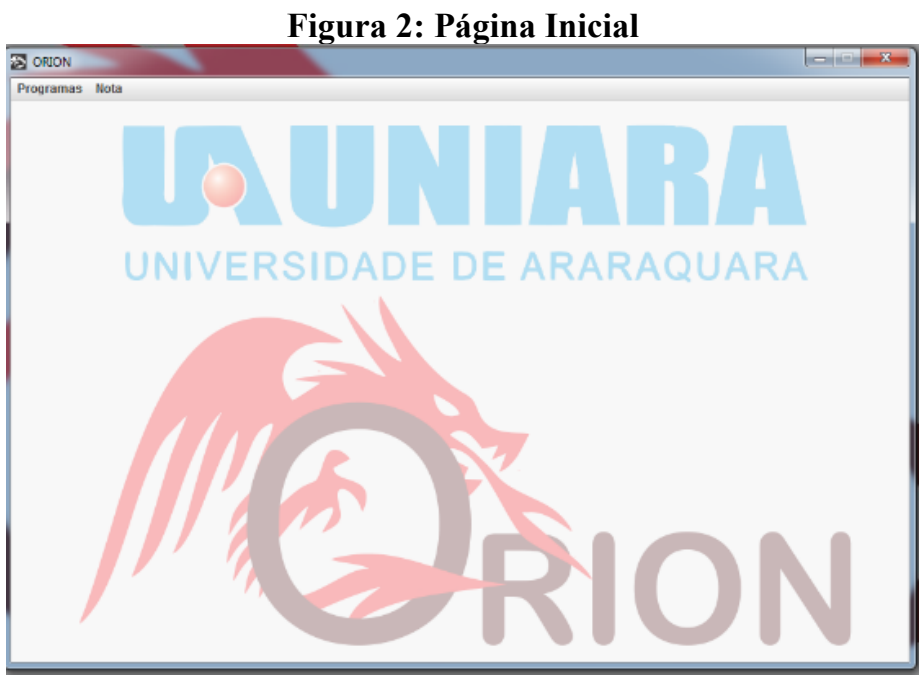

Fonte: Autor, 2020

\subsection{Inserção de dados}

A inserção de dados para as vigas é feita de forma simples, sendo necessário primeiramente as características básicas da viga. Figura 3.

Figura 3: Dados iniciais

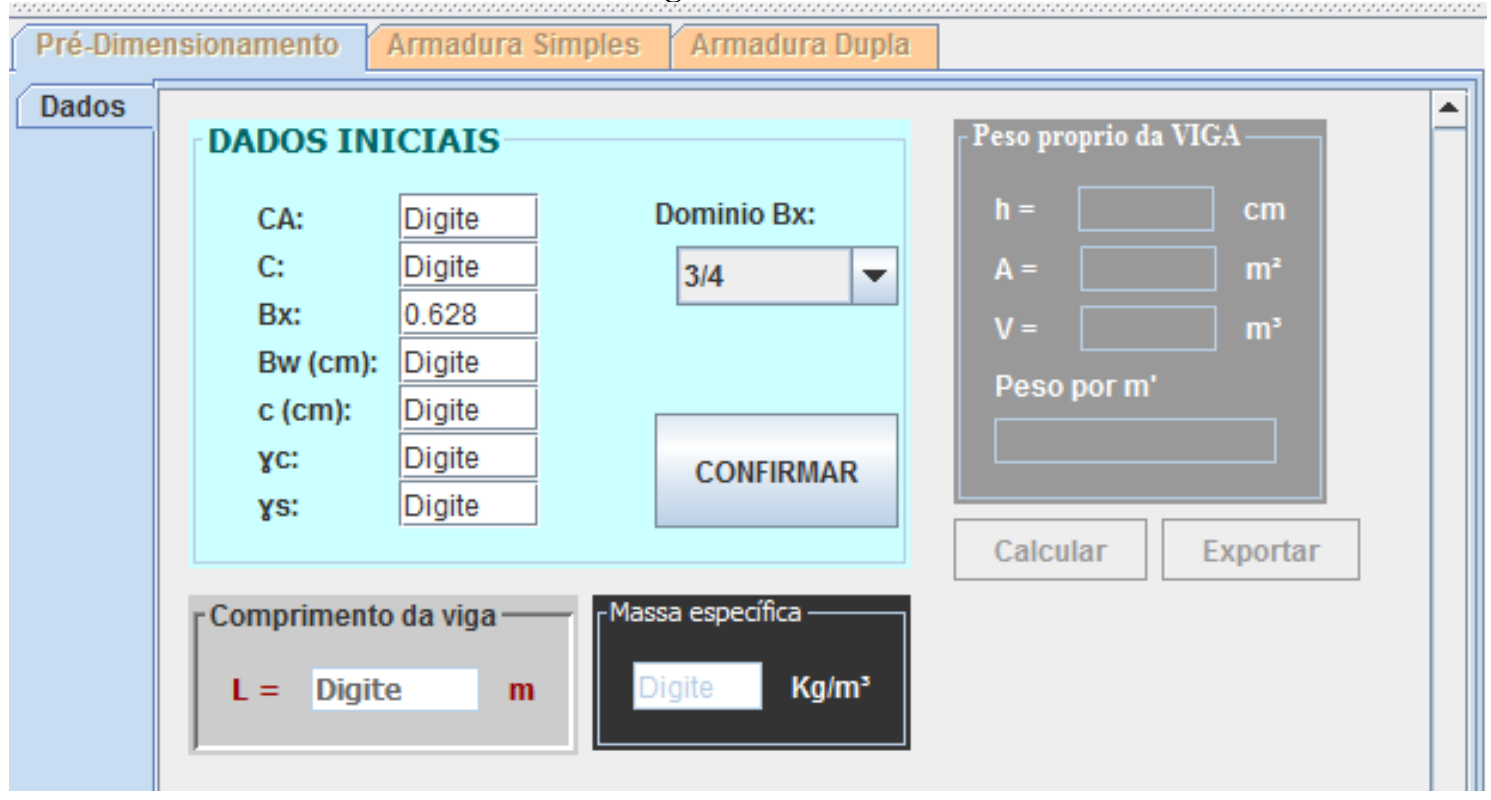

Fonte: Autor, 2020

Após a inserção dos dados iniciais é necessário clicar em Calcular para ser habilitado o console de desenho. 


\subsection{Saída de dados}

A apresentação dos dados e dos cálculos é basicamente apresentados da seguinte forma:

\section{a. Gráfico de esforço cortante e Momento Fletor. Figura 4.}

A plotagem dos gráficos é obtida utilizando o software VSOL publicado com a licença GNU General Public License version 3.0 (GPLv3), com uso restrito a fins educacionais, disponível no site sourceforge.net para obter uma classe do método dos elementos finitos, que é um procedimento numérico para determinar soluções aproximadas de problemas de valores sobre o contorno de equações diferenciais, será desenvolvido os gráficos de momento fletor e força cortante com a finalidade de encontrar o máximo valor para os cálculos. O esforço cortante é utilizado para os cálculos dos estribos posteriormente e o momento fletor para o cálculo das armaduras longitudinais.

Figura 4: Gráficos de esforços.

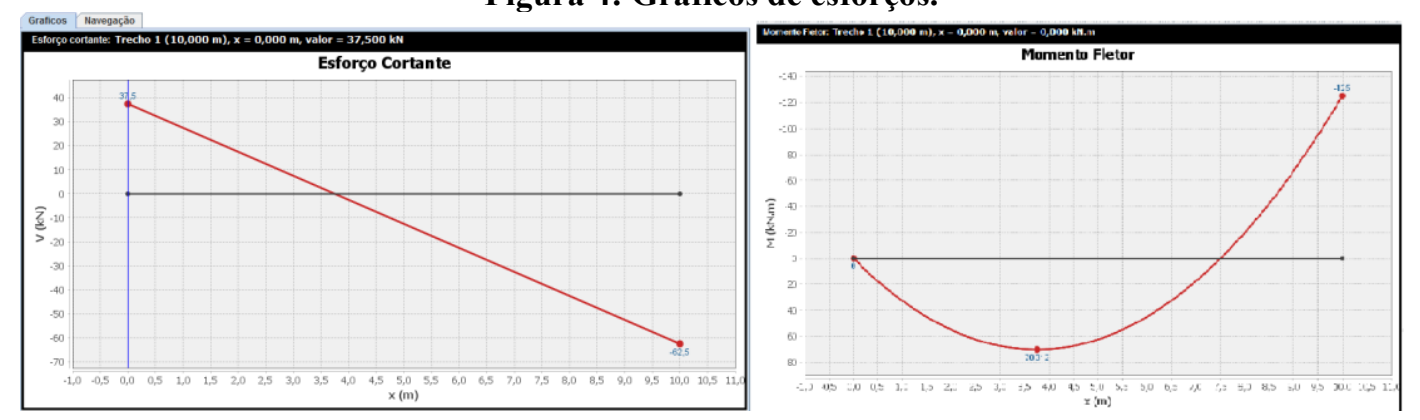

Fonte: Autor, 2020

\section{b. Armadura Longitudinal.}

A armadura longitudinal é calculada para vigas armadas de forma simples ou com armadura dupla, através do gráfico de momento fletor.

\section{c. Estribos}

Os estribos são calculados no modelo I e modelo II para vigas armadas de forma simples ou com armadura dupla, através do gráfico de esforço cortante. 


\section{EXEMPLO NUMÉRICO DE VIGA}

Nos próximos subitens serão construídos alguns exemplos numéricos para uma seção de concreto armado típica. Em todos os exemplos a geometria da seção transversal e a armação serão constantes.

Figura 5: Exemplo de cálculo de viga.

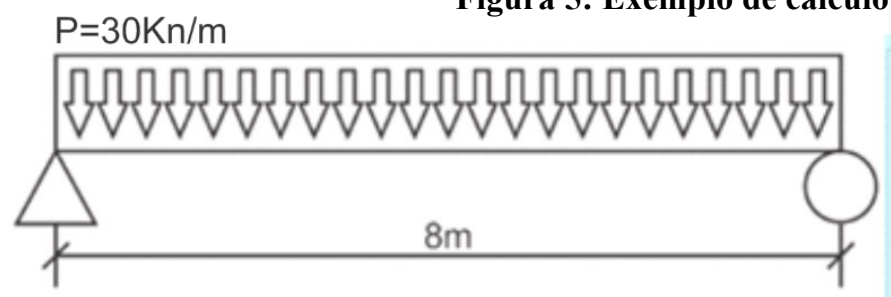

DADOS INICIAIS

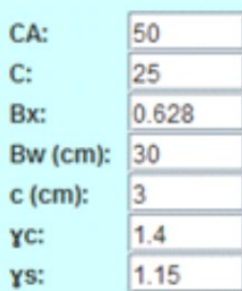

Dominio Bx:

Fonte: Autor, 2020

a. Pré-dimensionamento

$$
\begin{gathered}
h=\frac{8}{10}=0,8 \mathrm{~m} \\
\text { Vol }=0,3 * 0,8 * 8 \\
\text { Vol }=1,92 \mathrm{~m}^{3} \\
\frac{\text { Pviga }}{\mathrm{m}}=\gamma \mathrm{ca} * \mathrm{~A} \\
\frac{\text { Pviga }}{\mathrm{m}}=6 \frac{\mathrm{Kn}}{\mathrm{m}} \\
\text { Ptotal }=P+Q \\
\text { Ptotal }=30+6=36 \mathrm{Kn} / \mathrm{m} \\
\mathrm{m}=\frac{P * l^{2}}{8} \\
m=288 \mathrm{Kn} * \mathrm{~m}
\end{gathered}
$$

Figura 6: Momento fletor. 


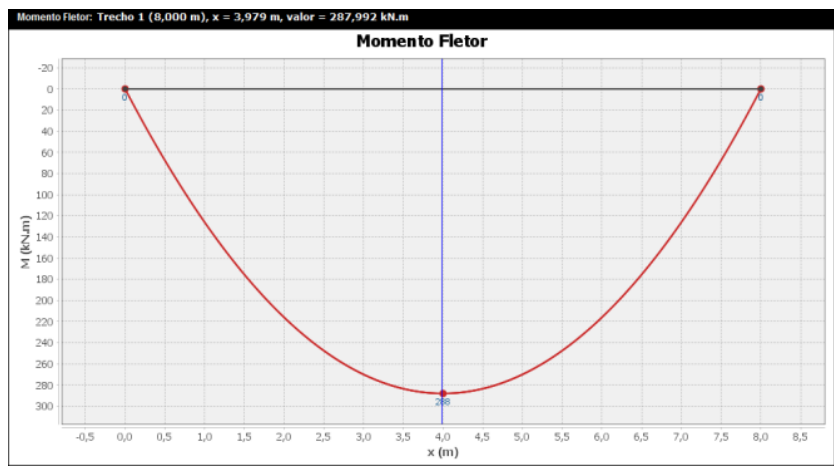

Fonte: Autor, 2020

b. Armadura Longitudinal Simples

$$
\begin{gathered}
d=\sqrt{\frac{m d}{0,68 * b w * b x * f c d *(1-0,4 * b x)}} \\
d=48,51 \mathrm{~cm} \\
A s=\frac{0,68 * b w * d * f c d * b x}{\sigma s} \\
A s=\frac{0,68 * 30 * 48,51 * \frac{2,5}{1,4} * 0,628}{\frac{50}{1,15}} \rightarrow A s=25,52 \mathrm{~cm}^{2}
\end{gathered}
$$

\section{c. Armadura Longitudinal Dupla}

Adotando $\mathrm{d}=50 \mathrm{~cm}$

$$
\begin{gathered}
k c=\frac{B w * d^{2}}{m d} \rightarrow k c=\frac{30 * 50^{2}}{288 * 1,4 * 100} \rightarrow k c=1,86 \\
b x=\frac{0.919117647059 *\left(1,36 * f c d * k c-\sqrt{1,8496 * f c d^{2} * k c^{2}-4,352 * f c d * k c}\right)}{f c d * k c} \\
k s=\frac{1}{\frac{C A}{\gamma S} *(1-0,4 * B x)} \rightarrow k s=\frac{1}{\frac{50}{1,15} *(1-0,4 * 0,5750)} \rightarrow k s=0,03
\end{gathered}
$$

\section{d. Estribos}




$$
\begin{aligned}
& F y w d=\frac{C A}{\gamma S}=\frac{50}{1,15}=43,478 \\
& F y w d(M P a)=\frac{0,21 * \sqrt[3]{F c k^{2}}}{\gamma c}=\frac{0,21 * \sqrt[3]{25^{2}}}{1.4}=1,282 \\
& \operatorname{Fywd}\left(K n / \mathrm{cm}^{2}\right)=\frac{\operatorname{Fctd}(M P a)}{10}=0,128 \\
& V s d=V k * \gamma c=144 * 1,4=201,6 \\
& \operatorname{Vrd} 2=0,27 *\left(1-\frac{C}{250}\right) * \frac{\frac{f c k}{10}}{1,14} * b w * d \\
& \operatorname{Vrd} 2=0,27 *\left(1-\frac{25}{250}\right) * \frac{\frac{25}{10}}{1,14} * 30 * 50 \rightarrow \operatorname{Vrd} 2=650,893 \\
& V s d \leq V r d 2 \rightarrow \text { Passa } \\
& V c=0,6 * F c t d\left(\frac{K n}{c m^{2}}\right) * b w * d \\
& V c=0,6 * 0,128 * 30 * 50 \rightarrow V c=115,423 \\
& V s w=V s d-V c \\
& V s w=201,6-115,423 \rightarrow V s w=86,177 \\
& \operatorname{Cotang}(a)=\frac{\operatorname{Cos}\left(a * \frac{\pi}{180}\right)}{\operatorname{Sen}\left(a * \frac{\pi}{180}\right)} \rightarrow \operatorname{Cotang}(a)=6,123 \\
& \operatorname{Cotang}(\varnothing)=\frac{\operatorname{Cos}\left(\varnothing * \frac{\pi}{180}\right)}{\operatorname{Sen}\left(\varnothing * \frac{\pi}{180}\right)} \rightarrow \operatorname{Cotang}(\varnothing)=1 \\
& a s w=\frac{v s w}{0,9 * d * f y w d} * s \\
& s=\frac{0,9 *\left(N^{\mathrm{o}} \text { Ramos }\right) * d * f y w d *(\operatorname{Cotang}(a)+\operatorname{Cotang}(\varnothing)) * \operatorname{seno}\left(a *\left(\frac{\pi}{180}\right)\right)}{v s w}
\end{aligned}
$$




$$
s=\frac{0,9 * 2 * 50 * 43,47826 *(6,123+1) * \operatorname{seno}\left(90 *\left(\frac{\pi}{180}\right)\right)}{86,177} s=8,90
$$

\section{e. Procedimento utilizado pelo software}

\begin{tabular}{|c|c|c|c|}
\hline No & Identificador & Tipo & Deslocamento \\
\hline 1 & U1 & Translação & Fixo \\
\hline 1 & U2 & Rotação & Livre \\
\hline 2 & U3 & Translação & Fixo \\
\hline 2 & U4 & Rotação & Livre \\
\hline
\end{tabular}

i. Matriz de rigidez de cada membro:

Quantidade de membros: 1

K 1 - Matriz de rigidez do membro $1(0,000 \mathrm{~m}$ a 8,000 m)

$$
\mathrm{L}=8,000 \mathrm{~m}
$$

\begin{tabular}{|c|c|c|c|c|}
\hline $\mathbf{U 1}$ & $\mathbf{U 2}$ & $\mathbf{U 3}$ & $\mathbf{U 4}$ & \\
\hline 0,0234 & 0,0938 & $-0,0234$ & 0,0938 & U1 \\
\hline 0,0938 & 0,5 & $-0,0938$ & 0,25 & U2 \\
\hline$-0,0234$ & $-0,0938$ & 0,0234 & $-0,0938$ & U3 \\
\hline 0,0938 & 0,25 & $-0,0938$ & 0,5 & U4 \\
\hline
\end{tabular}

ii. Matriz de rigidez global - K G :

\begin{tabular}{|c|c|c|c|c|}
\hline $\mathbf{U 1}$ & $\mathbf{U 2}$ & $\mathbf{U 3}$ & $\mathbf{U 4}$ & \\
\hline 0,0234 & 0,0938 & $-0,0234$ & 0,0938 & U1 \\
\hline 0,0938 & 0,5 & $-0,0938$ & 0,25 & U2 \\
\hline$-0,0234$ & $-0,0938$ & 0,0234 & $-0,0938$ & $\mathbf{U 3}$ \\
\hline 0,0938 & 0,25 & $-0,0938$ & 0,5 & U4 \\
\hline
\end{tabular}

iii. Matriz de deslocamentos livres - K D :

\begin{tabular}{|c|c|c|}
\hline U2 & U4 & \\
\hline 0,5 & 0,25 & U2 \\
\hline 0,25 & 0,5 & U4 \\
\hline
\end{tabular}

iv. Deslocamentos nodais - $\{D\}$ :

Considerando que $\{\mathrm{D}\}=[\mathrm{K} \mathrm{D}]^{-1} \mathrm{x}\{\mathrm{P}\}$ 
v. Matriz de deslocamentos livres inversa - [K D ]-1 :

\begin{tabular}{|c|c|c|}
\hline $\mathbf{U 2}$ & $\mathbf{U 4}$ & \\
\hline 2,6667 & $-1,3333$ & U2 \\
\hline$-1,3333$ & 2,6667 & $\mathbf{U 4}$ \\
\hline
\end{tabular}

vi. Matriz de reações - [K R ]:

\begin{tabular}{|c|c|c|}
\hline U2 & U4 & \\
\hline 0,0938 & 0,0938 & U1 \\
\hline$-0,093$ & $-0,0938$ & U3 \\
\hline
\end{tabular}

vii. Equações de cada membro:

Trecho $1(0,000 \mathrm{~m}$ a $8,000 \mathrm{~m})$

Esforço cortante $=V=-36 . x+144$

Momento fletor $=\mathrm{M}=-18 . \mathrm{x} 2+144 . \mathrm{x}$

\section{CONSIDERAÇÕES FINAIS}

Este trabalho apresentou a elaboração de um software desenvolvido para auxiliar o estudo de estruturas em concreto armado limitando-se ao cálculo de lajes e vigas para auxiliar na didática em sala de aula, o software traz de maneira lúdica e intuitiva a resolução de problemas complexos, a fim de despertar o interesse dos estudantes em buscar um conhecimento mais aprofundado sobre o assunto e, deste modo, tornar a engenharia civil mais atraente e minimizar os desastres causados pela falta de conhecimento por parte dos engenheiros, é importante observar que o software não substitui a teoria das obras de concreto, e seu conhecimento é essencial para o uso e entendimento das etapas de cálculos.

Utilizando a linguagem de programação java, foi desenvolvido um software livre e eficaz no cálculo e dimensionamento no âmbito do concreto armado, com a intenção de auxiliar os alunos na compreensão e aprendizagem dos cálculos estruturais, podendo ser implementado futuramente outras estruturas de concreto armado, como fundação e pilares, além de estruturas multidisciplinares como estruturas de madeiras e metálicas, atualmente o software está disponível em http://www.softwareorion.webnode.com, sendo focado em: 
- Realizar o pré-dimensionamento de vigas.

- Cálculo de esforços cortante e momento fletor em vigas.

- Cálculo de armadura simples Longitudinal (usando o momento fletor) e Estribos (usando o esforço cortante).

- Cálculo de armadura dupla Longitudinal (usando o momento fletor) e Estribos (usando o esforço cortante) o usuário definido a altura da viga, trabalhando fora do domínio 3/4.

- Cálculo de armaduras de lajes do Tipo 1 até o Tipo 6 utilizando o momento fletor.

- $\quad$ Cálculo de reações de apoios em lajes do Tipo 1 até o Tipo 6. 
REFERÊNCIAS

ABNT - Associação Brasileira de Normas Técnicas. NBR 6118: Projeto de estruturas de concreto - Procedimento. Rio de Janeiro, 2014.

AZEVEDO, Álvaro. MÉTODO DOS ELEMENTOS FINITOS. $1^{\text {a }}$ Edição. ed. Portugal: [s. $\quad$ n.], 2003. Disponível em: http://www.alvaroazevedo.com/publications/books/livro_mef_aa_1ed/doc/livro_mef_aa.pdf. Acesso em: 1 jun. 2020.

DEITEL, Paul; DEITEL, Harvey. Java: Como Programar. oitava. ed. [S. l.: s. n.], 1. sem 2010. 1145 p.

FERNANDES, Gilson B. SOLICITAÇÕES NORMAIS CÁLCULO NO ESTADO LIMITE ÚLTIMO. 2006. CAMPINAS.

PINHEIRO, Libânio M. et al. FUNDAMENTOS DO CONCRETO E PROJETO DE EDIFÍCIOS. 2010. 